hep-th/9909140

ETH-TH/99-25

ESI-759

September 1999

\title{
CONFORMAL BOUNDARY CONDITIONS AND THREE-DIMENSIONAL TOPOLOGICAL FIELD THEORY
}

\author{
Giovanni Felder, Jürg Fröhlich, Jürgen Fuchs and Christoph Schweigert \\ ETH Zürich \\ CH - 8093 Zürich
}

\begin{abstract}
We present a general construction of all correlation functions of a two-dimensional rational conformal field theory, for an arbitrary number of bulk and boundary fields and arbitrary topologies. The correlators are expressed in terms of Wilson graphs in a certain three-manifold, the connecting manifold. The amplitudes constructed this way can be shown to be modular invariant and to obey the correct factorization rules.
\end{abstract}


Two-dimensional conformal field theory plays a fundamental role in the theory of twodimensional critical systems of classical statistical mechanics [1], in quasi one-dimensional condensed matter physics [2] and in string theory [3]. The study of defects in systems of condensed matter physics [4], of percolation probabilities [5] and of (open) string perturbation theory in the background of certain string solitons, the so-called D-branes [6], forces one to analyze conformal field theories on surfaces that may have boundaries and / or can be non-orientable.

In this letter we present a new description of correlation functions of an arbitrary number of bulk and boundary fields on general surfaces. We also show how to compute various types of operator product coefficients from our formulas. For simplicity, in this letter we restrict our attention to boundary conditions that preserve all bulk symmetries. Moreover, we take the modular invariant torus partition function that encodes the spectrum of bulk fields of the theory to be given by charge conjugation. Technical details and complete proofs will appear in a separate publication.

Given a chiral conformal field theory, such as a chiral free boson, our aim is to compute correlation functions on a two-dimensional surface $X$ that may be non-orientable and can have a boundary. To this end, we first construct the so-called double $\hat{X}$ of the surface $X$. This is an oriented surface, on which an orientation reversing map $\sigma$ of order two acts in such a way that $X$ is obtained as the quotient of $\hat{X}$ by $\sigma$. Thus $\hat{X}$ is a two-fold cover of $X$; but this cover is branched over the boundary points, which correspond to fixed points of the map $\sigma$. For example, when $X$ is the disk $D$, then $\hat{X}$ is the two-sphere and $\sigma$ is the reflection about its equatorial plane. For $X$ the cross-cap, i.e. the real projective plane $\mathbb{R P}^{2}, \hat{X}$ is again the two-sphere, but $\sigma$ is now the antipodal map. Finally, when $X$ is closed and orientable, the double $\hat{X}$ consists of two disconnected copies of $X$ with opposite orientation, $\hat{X} \cong X \sqcup(-X)$.

Quite generally, correlation functions on a surface $X$ can be constructed from conformal blocks on its double $\hat{X}[\overline{7}, 8]$. As a first step, one has to find the pre-images on $\hat{X}$ of all insertion points on $X$, and associate a primary field of the chiral conformal field theory to each of them. Since bulk points have two pre-images, for a bulk field two chiral labels $j$ and $j^{*}$ are needed, corresponding to left and right movers. Boundary fields, in contrast, carry a single label $k$; yet, they should not be thought of as chiral objects.

Having associated these labels to the geometric data, we can assign a vector space of conformal blocks, not necessarily of dimension one, to every collection of bulk and boundary fields on $X$. The correlation function is one specific element in this space. This element must obey modular invariance and factorization properties. The conformal bootstrap programme [3] allows to determine the correlation function by imposing these properties as constraints. Fortunately, the connection between conformal field theory in two dimensions and topological field theory in three dimensions supplies us with a most direct way to construct concrete elements in the spaces of conformal blocks. What one must do in order to specify a a definite element in the space of conformal blocks is to find a three-manifold $M_{X}$ whose boundary is $\hat{X}$,

$$
\partial M_{X}=\hat{X}
$$

as well as a Wilson graph $W$ in $M_{X}$ that ends at the marked points on $\hat{X}$. This can be done for any arbitrary rational conformal field theory; for details, which are based on the axiomatization in [9], we refer to [10]. In the particular case of WZW models, Chern-Simons theory can be used [11, 12, 13] for this construction. For these models, the element in the space of conformal 
blocks is obtained by the Chern-Simons path integral

$$
\int \mathcal{D} A W \exp \left(\mathrm{i} \frac{k}{4 \pi} \int_{M_{X}} \operatorname{Tr}\left(A \wedge \mathrm{d} A+\frac{2}{3} A \wedge A \wedge A\right)\right)
$$

with appropriate parabolic conditions at the punctures.

Thus to obtain a correlation function on $X$, we first construct a certain three-manifold $M_{X}$ with boundary $\hat{X}$, which we call the connecting three-manifold. Technically, the manifold $M_{X}$ can be characterized as follows. When $X$ does not have a boundary, then $M_{X}=(\hat{X} \times[-1,1]) / \mathbb{Z}_{2}$, where the group $\mathbb{Z}_{2}$ acts on $\hat{X}$ by $\sigma$ and on the interval $[-1,1]$ by the sign flip $t \mapsto-t$ for $t \in[-1,1]$. Thus $M_{X}$ consists of pairs $(x, t)$ with $x$ a point on the double $\hat{X}$ and $t$ in $[-1,1]$, modulo the identification $(x, t) \sim(\sigma(x),-t)$. For fixed $x$, the points of the form $(x, t)$ form a segment, the connecting interval, joining the two pre-images of a point in $X$. When $X$ has a boundary, we obtain $M_{X}$ from $(\hat{X} \times[-1,1]) / \mathbb{Z}_{2}$ by contracting the connecting intervals over the boundary to single points, in such a way that $M_{X}$ remains a smooth manifold. (An equivalent construction, in which the boundary intervals are not contracted, was given in [13].)

It is readily checked that the boundary of the connecting manifold $M_{X}$ is indeed the double $\hat{X}$. Moreover, $M_{X}$ connects the two pre-images of a bulk point by an interval in such a manner that the connecting intervals for distinct bulk points do not intersect. Let us list a few examples. For a disk, the connecting manifold is a solid three-ball, and the connecting intervals are all perpendicular to the equatorial plane. Similarly, when $X$ is the annulus, $M_{X}$ is a solid torus. For $X$ the cross-cap, the connecting manifold $M_{X}$ is best characterized by the fact that when glueing to its boundary a solid ball, we obtain $S^{3} / \mathbb{Z}_{2} \cong \mathbb{R P}^{3}$, which coincides with the group manifold of the Lie group $\mathrm{SO}(3)$. For closed orientable surfaces $X$, the bundle $M_{X}$ is just the trivial bundle $X \times[-1,1]$; e.g. when $X$ is a sphere, then $M_{X}$ can be visualized as consisting of the points between two concentric spheres.

The next step is to specify a certain Wilson graph in $M_{X}$. The prescription, which is illustrated in figure 1 for the case of a disk with an arbitrary number of insertions in the bulk and on the boundary, is as follows. First, for every bulk insertion $j$, one joins the pre-images of the insertion point by a Wilson line running along the connecting interval. Next, one inserts one circular Wilson line parallel to each component of the boundary (a similar idea was presented in [13]) and joins every boundary insertion $k$ on the respective boundary component by a short Wilson line to the corresponding circular Wilson line. Moreover, the circular Wilson lines are required to run "close to the boundary", in the sense that none of the connecting intervals of the bulk fields passes between the circular Wilson lines and the boundary of $X$.

So far we have only specified the geometric information for the conformal blocks. To proceed, we also must attach a primary label of the chiral conformal field theory to each segment of the Wilson graph. For the bulk points, this prescription is immediate, as we are dealing with the charge conjugation modular invariant. Similarly, we are naturally provided with the labels $k$ for the short Wilson lines that connect the boundary insertions with the circular Wilson lines. In addition, the segments of the circular Wilson lines should encode the boundary conditions of the corresponding boundary segments. Recalling that those boundary conditions which preserve all bulk symmetries can be labelled by the primary fields of the chiral conformal field theory [14, we attach such a primary label $a$ to every segment of the circular Wilson lines. Finally, we must consider the three-valent junctions on the circular Wilson lines. For each of them we choose an element $\alpha$ in the space of chiral couplings between the label $k$ for the boundary field and 


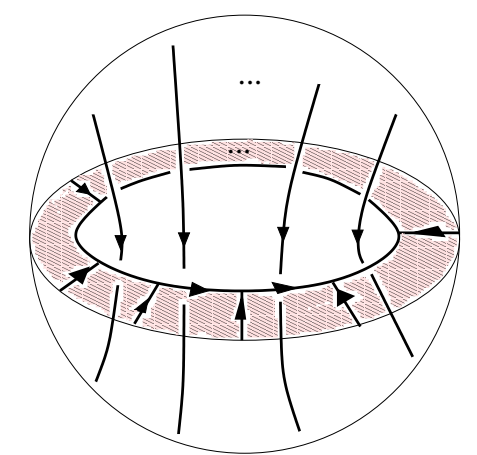

Figure 1: Wilson graph for the disk correlators

the two adjacent boundary conditions $a, b$. The dimension of this space of couplings is given by the fusion rules $\mathrm{N}_{k b}^{a}$ of the chiral theory. Indeed, it is known that boundary operators need an additional degeneracy label that takes its values in the space of chiral three-point blocks.

As a matter of fact, every segment of the Wilson graph should also be equipped with a framing [11] - in other words, we should not just specify a graph, but a ribbon graph. Moreover, the boundary $\hat{X}$ of $M_{X}$ must be endowed with additional structure, too. A careful discussion of these issues will be presented in [10]. As a side remark, we mention that the circular Wilson lines already come with a natural thickening to ribbons, which is obtained by connecting them to the pre-image of the boundary of $X$ in $\hat{X}$. (In figure 1 this is indicated by a shading.) Note that in the case of symmetry breaking boundary conditions [15] the labels of boundary fields and boundary conditions can be more general than in the bulk. This can be implemented in our picture, as the corresponding part of the graph with the circular Wilson line is disconnected from the rest of the Wilson graph.

Using appropriate surgery on three-manifolds, we can prove that the correlation functions obtained by our prescription possess the correct factorization (or sewing) properties and that they are invariant under large diffeomorphisms or, in more technical terms, under the relative modular group [16]. For a detailed account of these issues we refer to [10]. Here we restrict ourselves to the analysis of a few situations of particular interest; we also show how to recover known results for the structure constants from our formulas.

In our approach the structure constants are obtained as the coefficients that appear in the expansion of the specific element in the space of conformal blocks that represents a correlation function in a standard basis for the conformal blocks. For two points on the boundary of a solid three-ball such a standard basis is given by a Wilson line (with trivial framing) connecting the two points, while for three points one takes a Mercedes star shaped junction of three Wilson lines. Our general strategy for computing the coefficients is then to glue another three-manifold to the connecting manifold so as to obtain the partition function or, in mathematical terms, the link invariant, for a closed three-manifold. The values of such link invariants are available in the literature, see e.g. [11, 12, 9, 17].

Our first example is the correlator of two (bulk) fields on $S^{2}$, a closed and orientable surface. For the space of blocks to be non-zero, the two fields must be conjugate, i.e. carry labels $j$ and $j^{*}$, respectively. According to our prescription, the connecting manifold then consists of the filling between two concentric two-spheres, and the Wilson graph consists of two disjoint lines connecting the spheres, both labelled by $j$; this is depicted in figure 2. The space of conformal 
blocks for this situation is one-dimensional; its standard basis is displayed in figure 3. Thus the relevant three-manifold is given by the disconnected sum of two balls, each of which carries a single Wilson line. To both manifolds we glue two balls in which a Wilson line labelled by $j$ is running. In the case of the correlation function, the resulting manifold is a three-sphere with an unknot labelled by $j$, for which the value of the link invariant is $S_{0, j}$. ( $S$ is the modular Stransformation matrix of the chiral conformal field theory, and the label 0 refers to the vacuum primary field.) When applied to the manifold in figure 3, the glueing procedure produces two disjoint copies of $S^{3}$, each with an unknot labelled by $j$; the corresponding partition function is $S_{0, j}^{2}$. Comparing the two results we see that the two-point function on the sphere is expressed in terms of the standard basis as

$$
C\left(S^{2} ; j, j^{*}\right)=S_{0, j}^{-1} \cdot B\left(S^{2} ; j, j^{*}\right) \otimes B\left(-S^{2} ; j, j^{*}\right) .
$$

In other words, the normalization of the bulk fields $j$ differs by a factor of $\left(S_{0, j}\right)^{-1 / 2}$ from the more conventional prescription where they are 'canonically normalized to one'.

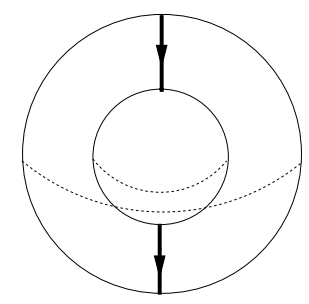

Figure 2: $C\left(S^{2} ; j, j^{*}\right)$

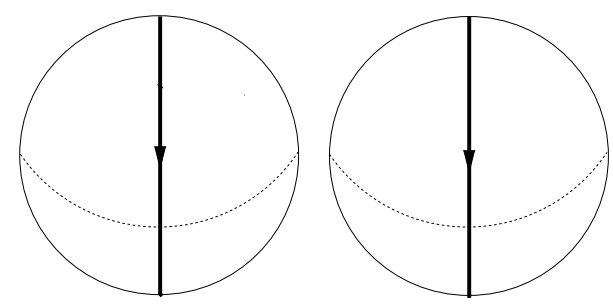

Figure 3: $B\left(S^{2} ; j, j^{*}\right) \otimes B\left(-S^{2} ; j, j^{*}\right)$

Next we discuss an example featuring an orientable surface with boundary; we compute the one-point amplitude for a bulk field $j$ on a disk $D$ with boundary condition $a$. Again the space of blocks is one-dimensional. Our task is then to compare the Wilson graph of figure $⿴$ with the standard basis that is displayed in figure 5. (In the present context, this particular conformal block is often called an 'Ishibashi state'). We now obtain the three-sphere by glueing with a single three-ball. When applied to the graph of figure 5, we get the unknot with label $j$ in $S^{3}$, for which the partition function is $S_{0, j}$. In the case of figure $⿴$ t we get a pair of linked Wilson lines with labels $a$ and $j$ in $S^{3}$; the value of the link invariant for this graph is $S_{a, j}$. Comparison thus shows that the correlation function is $S_{a, j} / S_{0, j}$ times the standard two-point block on the sphere,

$$
C\left(D_{a} ; j\right)=\left(S_{a, j} / S_{0, j}\right) \cdot B\left(S^{2} ; j, j^{*}\right) .
$$


Taking into account the normalization of the bulk fields as obtained in formula (3), we recover the known result that the correlator for a canonically normalized bulk field $j$ on a disk with boundary condition $a$ is $S_{a, j} / \sqrt{S_{0, j}}$ times the standard two-point block on the sphere. (This relation forms the basis of the so-called boundary state formalism [14].)

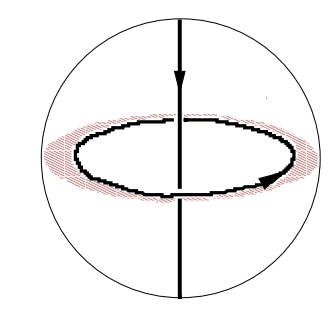

Figure 4: $C\left(D_{a} ; j\right)$

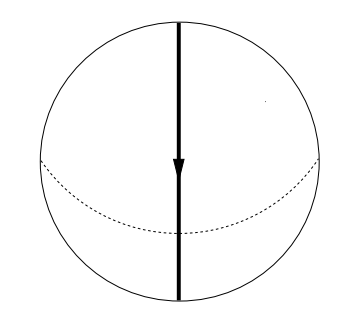

Figure 5: $B\left(S^{2} ; j, j^{*}\right)$

As a third example, we study again a one-point correlator of a (bulk) field $j$, now on the cross-cap $\mathbb{R}^{2}$, which does not have a boundary, but is non-orientable. The latter property forces us to be careful with the framing. The structure constants are obtained by comparing the correlator $C\left(\mathbb{R P}^{2} ; j\right)$ with the 'cross-cap state' $\psi_{j}$. This state is defined in figure 6; it is similar to the basis element $B\left(S^{2} ; j, j^{*}\right)$ of the two-point blocks on $S^{2}$, but now the Wilson line in the three-ball has a non-trivial framing, and accordingly in figure 6 we have drawn a ribbon instead of a line. A priori we could twist the line either by $+\pi$, thereby obtaining some state $\psi_{j}^{+}$, or by $-\pi$ and obtain another state $\psi_{j}^{-}$. These two vectors differ by a factor of $\mathrm{e}^{2 \pi \mathrm{i} \Delta_{j}}$, with $\Delta_{j}$ the conformal weight of $j$. Salomonically, we define the cross-cap state as

$$
\psi_{j}:=\mathrm{e}^{-\pi \mathrm{i} \Delta_{j}} \psi_{j}^{-}=\mathrm{e}^{\pi \mathrm{i} \Delta_{j}} \psi_{j}^{+} .
$$

Again the comparison of the correlator $C\left(\mathbb{R P}^{2} ; j\right)$ with the standard basis $\psi_{j}$ is carried out by glueing a three-ball with a Wilson line to the ball of figure 6. In contrast to the previous cases, however, this line is given a non-trivial framing; choosing the framing in such a way that the twist of the cross-cap state is undone, glueing the ball to the cross-cap state yields $S^{3}$ with the unknot, with partition function $Z\left(S^{3} ; j\right)=S_{0, j}$.

As already mentioned, glueing the three-ball to the connecting manifold of the cross-cap yields $\mathrm{SO}(3)$. It is also known that $\mathrm{SO}(3)$ can be obtained from $S^{3}$ by a surgery on the unknot with framing -2 . (Following how the framed graph is mapped by the surgery, one may visualize the situation as in figure 7.) Taking all framings properly into account, we obtain

$$
Z(\mathrm{SO}(3) ; j)=T_{0}^{1 / 2} \sum_{k} S_{0, k}\left(T_{k}\right)^{2} S_{k, j} T_{j}^{1 / 2}=P_{0, j}
$$


(with $T_{j} \equiv \mathrm{e}^{2 \pi \mathrm{i}\left(\Delta_{j}-c / 24\right)}$ ) for the invariant of this three-manifold, where in the second equality we expressed the result through the matrix 18 $P:=T^{1 / 2} S T^{2} S T^{1 / 2}$. We have thereby recovered the known formula

$$
C\left(\mathbb{R P}^{2} ; j\right)=\left(P_{0, j} / S_{0, j}\right) \cdot \psi_{j}
$$

for the one-point correlator on the cross-cap.

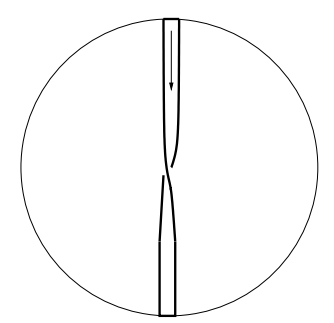

Figure 6: The state $\psi_{j}^{+}=\mathrm{e}^{-\pi \mathrm{i} \Delta_{j}} \psi_{j}$

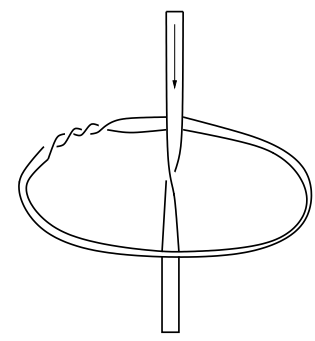

Figure 7 : A visualization of $C\left(\mathbb{R P}^{2} ; j\right)$

As a final example, consider three boundary fields $i, j, k$ on a disk. The relevant Wilson graph in the three-ball is of the type shown in figure 1, without any vertical Wilson lines along connecting intervals; it consists of a circular line (with segments labelled $a, b, c$ ) with three short Wilson lines (labelled $i, j, k$ ) attached to it. We must compare it to the standard basis for three-point blocks on the sphere, which is a Mercedes star shaped junction. This comparison can be made by performing a single fusing operation, followed by a contraction of the loop. For boundary fields, it is natural to define the correlation functions as linear forms on the degeneracy spaces for boundary operators. Denoting a basis of the degeneracy space for the boundary operator $\psi_{i}^{a c}$ by $\left\{e_{\alpha}\left[i c a^{*}\right]\right\}$, normalized by the quantum trace condition $\operatorname{tr}\left(e_{\alpha}\left[i c a^{*}\right] e_{\beta}\left[i^{*} a c^{*}\right]\right)=\delta_{\alpha, \beta}$, we find that

$$
C\left(D_{a, b, c} ; i, j, k\right)\left(e_{\alpha}\left[i c a^{*}\right] \otimes e_{\beta}\left[j a b^{*}\right] \otimes e_{\gamma}\left[k b c^{*}\right]\right)=\sum_{\kappa} \frac{S_{0,0}}{S_{k, 0}}\left\{\begin{array}{ccc}
i & c & a \\
b^{*} & j^{*} & k^{*}
\end{array}\right\}_{\gamma \kappa}^{\alpha \beta} e_{\kappa}[k j i],
$$

where the symbol $\left\{\begin{array}{ccc}i & j & k \\ l & m & n\end{array}\right\}_{\gamma \delta}^{\alpha \beta}$ denotes a fusing matrix (or quantum $6 j$-symbol) [17, 9].

One important conclusion we can draw from our results is that the construction of correlation functions from conformal blocks can be performed in a completely model-independent manner. All structure constants, for any arbitrary conformal field theory, can be expressed in terms of purely chiral data, such as conformal weights, the modular S-matrix, fusing matrices and 
the like. All specific properties of concrete models already enter at the chiral level. Physical quantities, such as the magnetization of an open spin chain or open string amplitudes in the background of D-branes, can be expressed in terms of the correlators studied in this letter.

\section{References}

[1] D.H. Friedan, Z. Qiu, and S.H. Shenker, Conformal invariance, unitarity, and critical exponents in two dimensions, Phys. Rev. Lett. 52 (1984) 1575

[2] I. Affleck, A current algebra approach to the Kondo effect, Nucl. Phys. B 336 (1990) 517

[3] A.A. Belavin, A.M. Polyakov, and A.B. Zamolodchikov, Infinite conformal symmetry in twodimensional quantum field theory, Nucl. Phys. B 241 (1984) 333

[4] M. Oshikawa and I. Affleck, Defect lines in the Ising model and boundary states on orbifolds, Phys. Rev. Lett. 77 (1996) 2604

[5] J.L. Cardy, Critical percolation in finite geometries, J. Phys. A 25 (1992) L201

[6] J. Polchinski, Dirichlet-branes and Ramond-Ramond charges, Phys. Rev. Lett. 75 (1995) 4724

[7] V. Alessandrini, A general approach to dual multiloop diagrams, Nuovo Cim. 2A (1971) 321

[8] J. Fuchs and C. Schweigert, Branes: from free fields to general backgrounds, Nucl. Phys. B 530 (1998) 99

[9] V.G. Turaev, Quantum Invariants of Knots and 3-Manifolds (de Gruyter, New York 1994)

[10] G. Felder, J. Fröhlich, J. Fuchs, and C. Schweigert, in preparation

[11] E. Witten, Quantum field theory and the Jones polynomial, Commun. Math. Phys. 121 (1989) 351

[12] J. Fröhlich and C. King, The Chern-Simons theory and knot polynomials, Commun. Math. Phys. 126 (1989) 167

[13] P. Hořava, Chern-Simons gauge theory on orbifolds: open strings from three dimensions, J. Geom. and Phys. 21 (1996) 1

[14] J.L. Cardy, Boundary conditions, fusion rules and the Verlinde formula, Nucl. Phys. B 324 (1989) 581

[15] J. Fuchs and C. Schweigert, Symmetry breaking boundaries I. General theory, preprint hepth/9902132, Nucl. Phys. B, in press; Symmetry breaking boundaries II. More structure, examples, preprint hep-th/9909025

[16] M. Bianchi and A. Sagnotti, Open strings and the relative modular group, Phys. Lett. B 231 (1989) 389

[17] G. Moore and N. Seiberg, Lectures on RCFT, in: Physics, Geometry, and Topology, H.C. Lee, ed. (Plenum Press, New York 1990), p. 263

[18] M. Bianchi and A. Sagnotti, The partition function of the SO(8192) bosonic string, Phys. Lett. B 211 (1988) 407 